\title{
Molecular cloning and functional characterization of a novel CBL-interacting protein kinase NtCIPK2 in the halophyte Nitraria tangutorum
}

\author{
L.L. Zheng*, Z. Gao*, J. Wang, H.R. Zhang and Y.C. Wang \\ Key Laboratory of Herbage \& Endemic Crop Biotechnology, \\ College of Life Sciences, Inner Mongolia University, Hohhot, China \\ *These authors contributed equally to this study. \\ Corresponding author: Y.C. Wang \\ E-mail: ycwang@imu.edu.cn / 13856265@qq.com
}

Genet. Mol. Res. 13 (3): 4716-4728 (2014)

Received April 5, 2013

Accepted September 12, 2013

Published July 2, 2014

DOI http://dx.doi.org/10.4238/2014.July.2.1

\begin{abstract}
CBL-interacting protein kinases (CIPKs) mediate many plant responses to abiotic stress. However, their functions are poorly understood in halophytes. In this study, we isolated a CIPK gene, NtCIPK2, from the halophyte Nitraria tangutorum. By sequence alignment and the construction of a phylogenetic tree, we found that NtCIPK2 is similar to CIPK2 proteins from other plants, and contains conserved domains and motifs. The promoter of NtCIPK2 harbors many cis-acting elements that might be recognized and bound by transcription factors that are related to hormones and stress responses. NtCIPK2 was ubiquitously and robustly expressed in all tested organs, and was induced by salinity, drought, heat, and cold stress. The overexpression of NtCIPK2 in Escherichia coli caused better growth against high salinity, alkalinity, and osmotic conditions, dehydration, and extreme temperatures (i.e., heat and cold) compared to the control.
\end{abstract}


Thus, NtCIPK2 is a candidate gene that might improve the stress tolerance of crops and herbs through genetic manipulation.

Key words: CIPK; Halophyte; Nitraria tangutorum; Stress tolerance

\section{INTRODUCTION}

Adverse environmental conditions, such as salt, drought, and extreme temperature, are ubiquitous in agricultural areas, negatively affecting the growth and yield of plants. To survive and complete their life cycle, plants have evolved a complex network of signal transduction pathways to cope with such stresses. As a universal second messenger, calcium is critical in the stress-signaling pathways (Knight, 2000). Most stresses elicit temporal and spatial changes in cytosolic free $\mathrm{Ca}^{2+}$ concentrations (Sanders et al., 2002). Stress-induced $\mathrm{Ca}^{2+}$ signals are decoded by $\mathrm{Ca}^{2+}$ sensors, and transduced through interactions with their protein kinases to regulate the expression of stress-responsive genes (Li et al., 2009).

Recently, a novel plant-specific calcineurin B-like protein (CBL)-interacting protein kinase (CIPK) network was dissected. CBL proteins contain 4 elongation factor hand domains for binding $\mathrm{Ca}^{2+}$ (Sánchez-Barrena et al., 2005), and associate with a group of serine/threonine kinases, designated as CIPKs (Kim et al., 2000). In general, CIPKs comprise a conserved Nterminal kinase domain with a putative activation loop between the DEF and APE residues (Batistic and Kudla, 2004) and a unique C-terminal-regulatory domain. A highly conserved FISL motif in the C-terminal domain of CIPKs mediates the CBL-CIPK interaction (Albrecht et al., 2001).

A database search revealed 26 CIPKs in the Arabidopsis genome and 30 CIPKs in the rice genome (Kolukisaoglu et al., 2004; Weinl and Kudla, 2009). Subsequent studies have demonstrated the functional conservation of the CBL-CIPK pathway in Populus (Yu et al., 2007), grape (Weinl and Kudla, 2009), sorghum (Li et al., 2010), and maize (Chen et al., 2011). Enhanced tolerance to diverse stresses has also been regularly observed in transgenic Arabidopsis because of the overexpression of CIPKs (Zhao et al., 2009; Chen et al., 2012), rice (Xiang et al., 2007), tomato (Wang et al., 2012; Huertas et al., 2012), tobacco (Tripathi et al., 2009), and apple (Hu et al., 2012).

Remarkable progress has been made in determining the functions of CIPKs in response to abiotic stress in model plants and certain crops (Imamura et al., 2008; Zhao et al., 2009; Tai et al., 2013). For example, in Arabidopsis, CIPK1 and CIPK3 are crosstalk nodes between the ABA-dependent and ABA-independent pathways under environmental stress (Kim et al., 2003; D'Angelo et al., 2006). CIPK6 is required for development and salt tolerance (Tripathi et al., 2009), while CIPK7 is involved in cold responses (Huang et al., 2011). The ectopic expression of $C I P K 8$ exhibits resistance to high glucose concentrations (Gong et al., 2002). CIPK9 and CIPK23 activate $\mathrm{K}^{+}$channels or transporters, and regulate $\mathrm{K}^{+}$uptake under low $\mathrm{K}^{+}$stress (Xu et al., 2006; Pandey et al., 2007; Liu et al., 2013). SOS2/CIPK24 is critical for salt tolerance, maintaining $\mathrm{Na}^{+}$and $\mathrm{Ca}^{2+}$ homeostasis (Cheng et al., 2004; Qiu et al., 2004). Yet, there is a paucity of reports about the function of CIPKs in other plants, particularly halophytes (Li et al., 2012).

Nitraria tangutorum Bobr. is a shrub that belongs to the Nitratia genus in the $\mathrm{Zy}$ gophyllaceae family. This species is a typical and native desert halophyte that grows under 
extreme conditions in northwest China. $N$. tangutorum seeds were collected from the Eastern Alxa-Western Ordos area $\left(106^{\circ} 27 \mathrm{E}-111^{\circ} 28^{\prime} \mathrm{E}, 39^{\circ} 13^{\prime} \mathrm{N}-40^{\circ} 52^{\prime} \mathrm{N}\right.$, elevation $1500-2100 \mathrm{~m}$ above sea level), which is a salinized desert in Inner Mongolia. This desert is characterized by a strong continental, weak monsoon, and arid or semiarid climate, with an average annual precipitation of $140.9-302.2 \mathrm{~mm}$ and an average annual temperature of $6.0^{\circ}-9.2^{\circ} \mathrm{C}$. The mean soil salinity and $\mathrm{pH}$ in this area are $0.7 \%$ and 9.0 , respectively $(\mathrm{Li}, 1990)$.

$N$. tangutorum has a vital ecological function because of its superior tolerance to salinity, alkalinity, drought, wind, and dust (Zheng et al., 2011). In addition, the berries of $N$. tangutorum are used as a nutritional food source and traditional herb by villagers for the treatment of hypertension, abnormal menstruation, and indigestion. These berries also decrease blood lipid levels and have antioxidative effects (Wang et al., 2007). As an important species that has ecological and economic value in a high-salinity and arid zone, identifying the tolerance-related genes of $N$. tangutorum would be beneficial for crop improvement. However, most research on $N$. tangutorum has been restricted to its physiological responses to stress environments. For instance, enhanced proline accumulation and elevated levels of calcium in $N$. tangutorum under $\mathrm{NaCl}$ treatment have been reported by Yang et al. (2010, 2013). In contrast, little is known about the molecular mechanisms underlying the perception and adaptability of $N$. tangutorum to various stressors.

In this study, a novel CIPK gene (NtCIPK2) was isolated from the halophytic shrub $N$. tangutorum by the rapid amplification of cDNA end (RACE) cloning. In addition, the $N t C I P K 2$ promoter was isolated and analyzed. The expression of NtP5CS was upregulated by various abiotic stresses. Its function in tolerance to high salinity, alkalinity, and osmotic conditions, dehydration (drought), and extreme temperatures (hot and cold) was also examined in Escherichia coli.

\section{MATERIAL AND METHODS}

\section{Plant growth and stress treatment}

Seeds were sown and grown in a growth chamber at $25^{\circ} \mathrm{C}$ with a 12 -h light/dark period and relative air humidity of 50\%. After 2 months of culture, the young leaves were used for cloning and various organs (root, shoot and leaves) were used for gene expression assays. In addition, seedlings were treated with $200 \mathrm{mM}$ sodium chloride and $10 \%$ polyethylene gly$\operatorname{col}(\mathrm{PEG})$, then subject to either $50^{\circ}$ or $4^{\circ} \mathrm{C}$ for $6 \mathrm{~h}$. Subsequently, the leaves were collected to analyze the responses of $N t C I P K 2$ to abiotic stress.

\section{Isolation of total RNA and synthesis of first-strand cDNA}

Total RNA was extracted using the RNAiso Kit (Takara, Dalian, China), following manufacturer protocols, and treated with deoxyribonuclease, before the reverse transcription (RT) reaction. One microgram of total RNA was used for cDNA synthesis. First-strand cDNA was synthesized using the M-MLV Reverse Transcriptase Kit (for semi-quantitative PCR; Promega, USA) or the SMARTer ${ }^{\mathrm{TM}} R A C E$ cDNA Amplification Kit (for RACE; Clontech, USA). The cDNA was diluted with nuclease-free water to obtain a total volume of 20 $\mathrm{ng} / \mu \mathrm{L}$. 


\section{Cloning of full-length NtCIPK 2 cDNA}

Degenerate primers (DECIPKF and DECIPKR; Table 1) were designed based on the alignment of all known amino acid sequences of AtCIPK members. PCR was performed in a $25-\mu \mathrm{L}$ mixture, containing $20 \mathrm{ng}$ template cDNA, $1.0 \mu \mathrm{M}$ of each primer, $200 \mu \mathrm{M}$ dNTPs, and 1.0 U TransStart ${ }^{\mathrm{TM}}$ Taq DNA polymerase (TransGen, China). DNA amplification was completed at $94^{\circ} \mathrm{C}$ for $5 \mathrm{~min}, 35$ cycles at $94^{\circ} \mathrm{C}$ for $30 \mathrm{~s}, 50^{\circ} \mathrm{C}$ for $30 \mathrm{~s}$, and $72^{\circ} \mathrm{C}$ for $30 \mathrm{~s}$, followed by a final step at $72^{\circ} \mathrm{C}$ for $10 \mathrm{~min}$. The amplicon was gel-purified, cloned into pMD19-T (Takara), and sequenced (Sangon, China).

\begin{tabular}{|c|c|c|}
\hline Experiment & Primer name & Primer sequence $\left(5^{\prime}-3^{\prime}\right)$ \\
\hline \multirow[t]{2}{*}{$N t C I P K 2$ degenerate PCR } & DEF & CACCGTGA(T/C)CTAAA(A/G)CCAG \\
\hline & DER & TGACCCC(G/C)CAAGACCA(A/G)ATA \\
\hline \multirow[t]{5}{*}{ 5'- and 3'-RACE } & GSP5 & GGGAGCAACATAGGCTGGGGTTC \\
\hline & GSP3 & GGGTTGAGCGCTCTCTCTGATTCG \\
\hline & UPM (long) & CTAATACGACTCACTATAGGGCAAGC \\
\hline & & AGTGGTATCAACGCAGAGT \\
\hline & UPM (short) & CTAATACGACTCACTATAGGGC \\
\hline \multirow[t]{2}{*}{ Amplification of the complete coding region of $N t C I P K 2$} & CDSF & ATGATGGAACACAAAGGGAAAG \\
\hline & CDSR & CTAATGGTAATGCTGTAGCCGAG \\
\hline \multirow[t]{5}{*}{ Isolation of the $N t C I P K 2$ promoter } & SFP1 & CACGACACGCTACTCAACAC \\
\hline & SFP2 & ACTCAACACACCACCTCGCACAGC \\
\hline & CIPKP1 & CTTGGTCTTGGTGGCCATGACCTC \\
\hline & CIPKP2 & CCTTAACTGCTACACTCTGGCTGG \\
\hline & CIPKP3 & CCTTAGCGAAGGTCCCCTGGCCCA \\
\hline \multirow[t]{4}{*}{ Semiquantitative PCR } & CIPK2F & ACGACACTTCCTGCCTCAAATA \\
\hline & CIPK2R & CCCAAACAATGTCCTCCAGAGC \\
\hline & ACTINF & GGAATCCACGAGACCACCTACA \\
\hline & ACTINR & GATTGATCCTCCGATCCAGACA \\
\hline \multirow{2}{*}{ Construction of $N t C I P K 2$ expression vector } & NTCMQF & CGCGGATCCATGATGGAACAC \\
\hline & NTCMQR & ACGCGTCGACCTAATGGTAATG \\
\hline
\end{tabular}

Sequences underlined indicate restriction enzyme sites.

The sequence was analyzed by BLAST, and gene-specific primers (Table 1) were designed for 5'- and 3'-RACE. RACE was performed using the SMARTer ${ }^{\mathrm{TM}} R A C E$ cDNA Amplification Kit, following manufacturer protocols, in a $25-\mu \mathrm{L}$ mixture, containing $20 \mathrm{ng} 5$ 'or 3'-RACE cDNA, $1.0 \mu \mathrm{M}$ GSP5 or GSP3, $0.04 \mu \mathrm{M}$ long UPM, $0.2 \mu \mathrm{M}$ short UPM, $200 \mu \mathrm{M}$ dNTPs, and $1.0 \mathrm{U}$ Taq DNA polymerase. DNA was amplified at $94^{\circ} \mathrm{C}$ for $30 \mathrm{~s}, 68^{\circ} \mathrm{C}$ for $30 \mathrm{~s}$, and $72^{\circ} \mathrm{C}$ for $3 \mathrm{~min}$ for 30 cycles, followed by an extension at $72^{\circ} \mathrm{C}$ for $10 \mathrm{~min}$.

The 5'- and 3'-RACE products were extracted, inserted into the pMD19-T vector, and analyzed for sequence. After assembly of the fragments, the complete coding region of the cDNA was amplified with the specific primer pairs in Table 1. The PCR program was as follows: an initial denaturation step at $94^{\circ} \mathrm{C}$ for $5 \mathrm{~min}$, followed by 30 cycles at $94^{\circ} \mathrm{C}$ for $30 \mathrm{~s}, 55^{\circ} \mathrm{C}$ for $30 \mathrm{~s}$, and $72^{\circ} \mathrm{C}$ for $1.5 \mathrm{~min}$, and a final extension at $72^{\circ} \mathrm{C}$ for $10 \mathrm{~min}$. This gene was designated as $N t C I P K 2$.

\section{In silico analysis of $\mathrm{NtCIPK2}$}

The BLAST program (NCBI) was used to verify CIPK2 protein homology. Multiplesequence alignment was performed using Clustal X. Conserved domains of NtCIPK2 were 
predicted using Prosite (http://au.expasy.org/tools), and a phylogenetic tree of CIPK2 was constructed with the amino acid sequences of NtCIPK2 and Arabidopsis CIPK proteins by the neighbor-joining algorithm in MEGA 4.0, with 1000 bootstrap replications. Theoretical isoelectric points and mass values for the proteins were predicted by ExPASy ProtParam (http:// web.expasy.org/protparam). A structural search for NtCIPK2 was performed by TMHMM2.0 (http://www.cbs.dtu.dk/services/THHMM-2.0).

\section{Isolation of $\mathrm{NtCIPK2}$ promoter}

Genomic DNA was prepared from N. tangutorum seedlings using the Plant Genomic DNA Kit (Tiangen, China). Approximately $0.1 \mathrm{~g}$ of the sample was ground to a fine powder in liquid nitrogen, and the NtCIPK2 promoter was isolated by SiteFinding PCR, as previously described (Tan et al., 2005). PLACE (http://www.dna.affrc.go.jp/PLACE/signalup.html) and PlantCARE (http://bioinformatics.psb.ugent.be/webtools/plantcare/html) were used to analyze cis-regulatory elements.

\section{Analysis of expression patterns by semi-quantitative PCR}

Semi-quantitative PCR was performed to analyze the expression profile of NtCIPK2. $\beta$-actin (GenBank accession No. AB617805) was used as the reference gene. The 25- $\mu \mathrm{L}$ PCR product contained $20 \mathrm{ng}$ cDNA, $1.0 \mu \mathrm{M}$ of each primer, $200 \mu \mathrm{M}$ dNTPs, and 1.0 U Taq DNA polymerase. PCR was performed at $94^{\circ} \mathrm{C}$ for $1 \mathrm{~min}$, followed by 28 cycles at $94^{\circ} \mathrm{C}$ for $20 \mathrm{~s}$, $55^{\circ} \mathrm{C}$ for $20 \mathrm{~s}$, and $72^{\circ} \mathrm{C}$ for $20 \mathrm{~s}$. The $N t C I P K 2$-specific and $\beta$-actin primers are listed in Table 1. The experiment was repeated in 3 separate times.

\section{Construction of $\mathrm{NtCIPK} 2$ expression vector and heterologous protein expression}

ORF primers with restriction enzyme sites for BamHI and SalI were designed (Table 1) based on the CIPK2 sequence of $N$. tangutorum. The amplified products were cloned into pGEX 4T1 at the BamHI-SalI site to express the CIPK2 protein that was fused with glutathione S-transferase (GST) at the N-terminus. Recombinant plasmids were transformed into E. coli Rosetta (DE3) cells. Transformants that harbored pGEX 4T1-NtCIPK2 were designated as RO/ NtCIPK2, while those with pGEX 4T1 were named as RO/pGEX and used as the control.

$\mathrm{RO} / \mathrm{NtCIPK} 2$ and control cells were cultured overnight in LB (Luria-Bertani) with $100 \mu \mathrm{g} / \mathrm{mL}$ ampicillin on a shaker at $37^{\circ} \mathrm{C}$. Fresh LB medium was inoculated with $1 \%$ volume of the overnight culture until $\mathrm{A}_{600}$ reached 0.6. Isopropyl $\beta$-D-thiogalactopyranoside (IPTG) was added to a final concentration of $1 \mathrm{mM}$ to induce the expression of $N t C I P K 2$, and all cells were grown at $30^{\circ} \mathrm{C}$ for another $4 \mathrm{~h}$. Next, the bacteria were pelleted, sonicated, diluted 2-fold in sample buffer, and loaded onto a $12 \%$ SDS-PAGE gel for analysis.

\section{Functional validation of $N t C I P K 2$ in $E$. coli under various abiotic stresses}

To evaluate the protective properties of the NtCIPK2 fusion protein, the effects of salt (400 $\mathrm{mM} \mathrm{NaCl})$, osmosis (1 M sorbitol), drought (10\% PEG), alkali (150 $\mathrm{mM} \mathrm{NaHCO}_{3}$, $\mathrm{pH} 9.0)$, heat $\left(50^{\circ} \mathrm{C}\right)$, and cold $\left(-20^{\circ} \mathrm{C}\right)$ on the growth of RO/NtCIPK2 and control cells were 
examined. Transformed E. coli cells were grown as described in the previous section. The original $\mathrm{A}_{600}$ values of the samples were adjusted to 1.0, and $100 \mu \mathrm{L}$ cells was added to $10 \mathrm{~mL}$ LB containing $\mathrm{NaCl}$, sorbitol, $\mathrm{PEG}$, or $\mathrm{NaHCO}_{3}$. Separately, $100 \mu \mathrm{L}$ cells was incubated at $50^{\circ} \mathrm{C}$ for $6 \mathrm{~h}$ or $-20^{\circ} \mathrm{C}$ for 3 days, and added to $10 \mathrm{~mL} \mathrm{LB}$ liquid medium.

The bacterial suspension was harvested every $2 \mathrm{~h}$ for $12 \mathrm{~h}$, and $\mathrm{A}_{600}$ was measured. All experiments were repeated 3 times with 3 replicates each time. The results are reported as means \pm standard deviation (SD). Statistical analysis was performed using one-way analysis of variance (ANOVA) with SPSS 19.0. A P value $<0.05$ indicated a significant difference.

\section{RESULTS}

\section{Cloning and characterization of CIPK2 cDNA from N. tangutorum}

The NtCIPK2 cDNA is 2049 nucleotides long with a 5'-UTR of 437 nucleotides, a predicted ORF of 1344 nucleotides, and a 3'-UTR of 268 nucleotides. The predicted ORF encodes a protein of 447 amino acid residues with a theoretical molecular mass of $50.64 \mathrm{kDa}$. The $\mathrm{pI}$ of this protein is 8.80 . Our BLAST demonstrated that NtCIPK2 shared high similarity with plant CIPKs. AtCIPK2 is its ortholog among the 26 Arabidopsis CIPK members, showing $67 \%$ sequence identity. Thus, NtCIPK2 cDNA was deposited into the GenBank (accession No. KC823044) as a novel member of the plant $C I P K$ family.

By multiple alignment analysis, the deduced NtCIPK2 sequence contained 2 domains: an N-terminal kinase domain with an activation loop and a C-terminal-regulatory domain with a CBL-interacting FISL motif (Figures 1 and 2). Ser 156, Thr 168, and Tyr 175, which are conserved in AtSOS2 and other CIPKs, were also observed in NtCIPK2 (Figure 1). As in AtCIPK2, there is no transmembrane helix domain in NtCIPK2, according to the prediction by TMHMM 2.0.

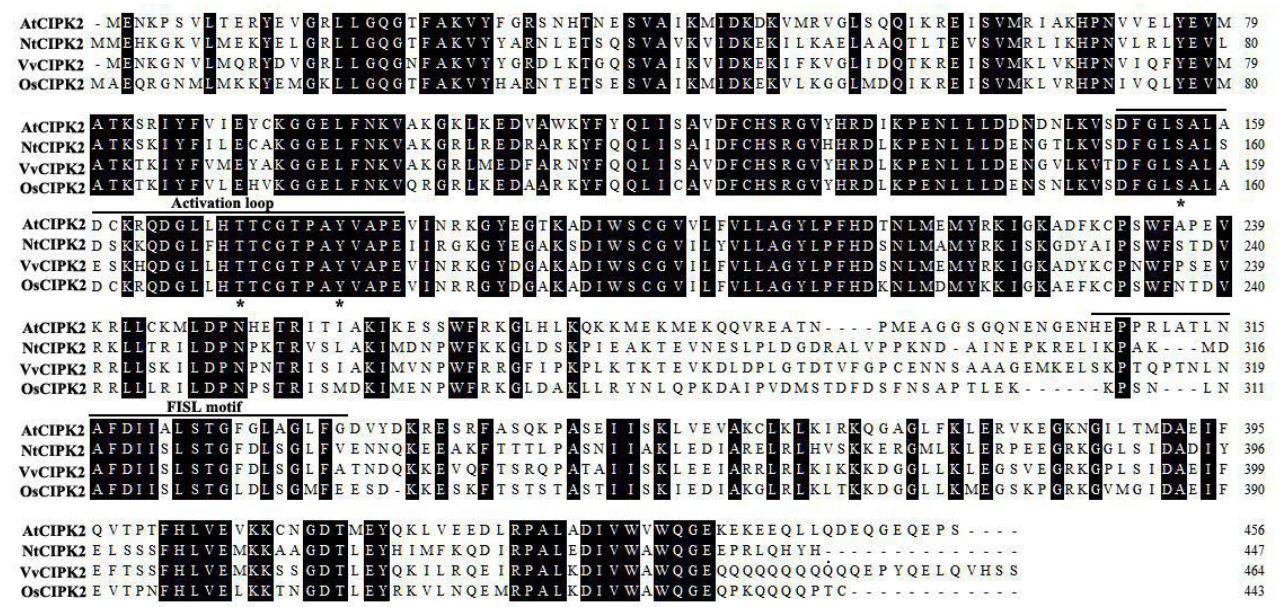

Figure 1. Deduced amino acid sequence alignment of NtCIPK2; peptide alignment of NtCIPK2 with homologs from Arabidopsis (AtCIPK2, AAF86506), Vitis vinifera (VvCIPK2, XP_002279331), and rice (OsCIPK2, ACD76974). Black boxes indicate identical residues; sequences under the dark line are the activation loop and FISL motif, respectively; three highly conserved residues (Ser, Tyr, and Thr) are marked with an asterisk in the activation loop. 


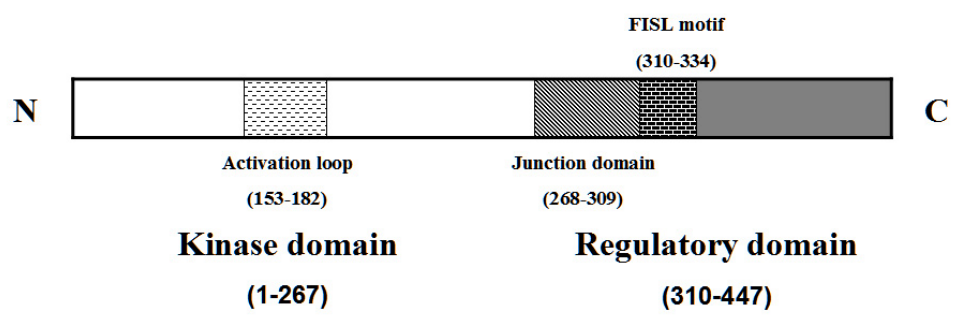

Figure 2. Schematic of the domain structure of NtCIPK2. The positions of the domains were determined based on comparison of the complete sequence of NtCIPK2 with the consensus sequence of all Arabidopsis CIPKs.

In the phylogenetic tree of the deduced amino acid sequences of NtCIPK2 and the Arabidopsis CIPK family, NtCIPK2 was closer to AtCIPK2 than AtSOS2, and is clustered into the subgroup without introns (Figure 3). We confirmed that NtCIPK2 did not have any introns in the $N$. tangutorum genome by amplifying it from cDNA and genomic DNA (data not shown).

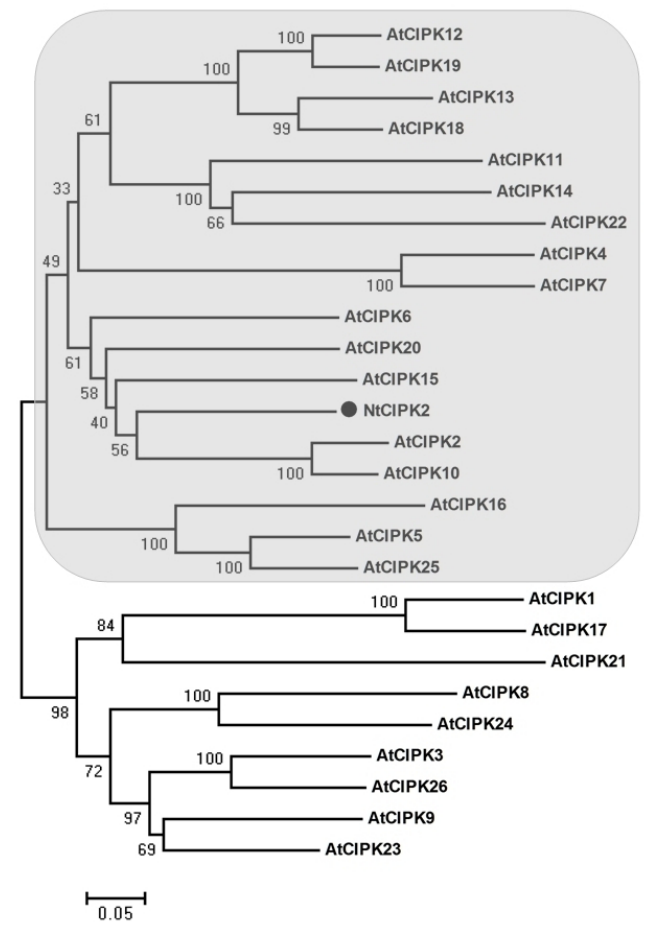

Figure 3. Phylogenetic tree of CIPK proteins. The tree was generated with NtCIPK2 and Arabidopsis CIPK proteins using MEGA 4.0, with 1000 bootstrap replications; the subgroup of CIPKs without introns is shownin the gray background; the Genbank accession numbers of the 26 AtCIPK proteins are: AtCIPK1, AAG28776; AtCIPK2, AAF86506; AtCIPK3, AAF86507; AtCIPK4, AAG01367; AtCIPK5, AAF86504; AtCIPK6, AAF86505; AtCIPK7, AAK16682; AtCIPK8, AAK16683; AtCIPK9, AAK16684; AtCIPK10, AAK16685; AtCIPK11, AAK16686; AtCIPK12, AAK16687; AtCIPK13, AAK16688; AtCIPK14, AAK16689; AtCIPK15, AAK16692; AtCIPK16, AAK50348; AtCIPK17, AAK64513; AtCIPK18, AAK59695; AtCIPK19, AAK50347; AtCIPK20, AAK61493; AtCIPK21, AAK59696; AtCIPK22, AAL47845; AtCIPK23, AAK61494; AtCIPK24, AAK72257; AtCIPK25, AAL41008; AtCIPK26, NP_850861. 


\section{Promoter analysis}

To identify putative cis-regulatory elements, approximately $1600 \mathrm{bp}$ of the upstream sequence from the start codon of NtCIPK2 was isolated and analyzed. Most elements in the NtCIPK 2 promoter were classified into 3 groups by function: light-responsive elements, phytohormone-responsive elements, and stress-related elements (Table 2).

Table 2. Prediction of stress response cis-acting elements in the NtCIPK2 promoter.

\begin{tabular}{|c|c|c|c|}
\hline Motif name & Sequences & Sites & Function \\
\hline ABRE & ACGTG & $\begin{array}{l}150(+) ; 1160(+) ; 1212(+) \\
1249(+) ; 1159(-)\end{array}$ & Abscisic acid responsiveness \\
\hline GARE & TCTGTTG & $282(+)$ & Gibberellin-responsive element \\
\hline GATA Box & GATA & $1477(+) ; 164(-) ; 1182(-)$ & Light-regulated and tissue-specific expression \\
\hline G-Box & SACGTB & $1159(+) ; 1159(-)$ & Light responsiveness \\
\hline GT1 & GRWAAW & $190(+) ; 194(-) ; 202(-) ; 168(-) ; 234(-)$ & Salt- and pathogen-induced gene expression \\
\hline HSE & AAAAAATTTC & $1060(+) ; 1062(-)$ & Heat stress responsiveness \\
\hline LTRE & CCGAAA & $186(-)$ & Low-temperature responsiveness \\
\hline MYB & CNGTTR & $284(+) ; 110(-) ; 122(-) ; 1039(-)$ & Dehydration responsiveness \\
\hline MYC & CANNTG & $\begin{array}{l}481(+) ; 720(+) ; 840(+) ; 933(+) \\
1096(+) ; 1125(+) ; 1145(+) ; \\
1159(+) ; 1227(+) ; 1438(+) ; \\
\text { 481(-); 720(-); 840(-); } 933(-) ; \\
\text { 1096(-);1125(-);1145(-); } \\
1159(-) ; 1227(-) ; 1438(-)\end{array}$ & $\begin{array}{l}\text { Multiple stresses (cold, dehydration, freezing, } \\
\text { ABA, etc.) responsiveness }\end{array}$ \\
\hline W-Box & TCAC & $742(+) ; 1487(+) ; 848(-)$ & Salicylic acid, wound, and pathogen responsiveness \\
\hline
\end{tabular}

\section{Expression analysis}

\section{Expression patterns of NtCIPK2 in vivo}

$N t C I P K 2$ transcripts were identified in all of the organs that we tested, including the root, shoot, and leaves, but varied between organs. For example, the shoot generated the least NtCIPK2 transcripts. NtCIPK2 was upregulated by salinity, drought, heat, and cold stress, with the highest induction being observed for salt and drought stress compared to the other treatments (Figure 4). Our data demonstrated that NtCIPK2 is involved in multiple stress-responsive pathways.

A

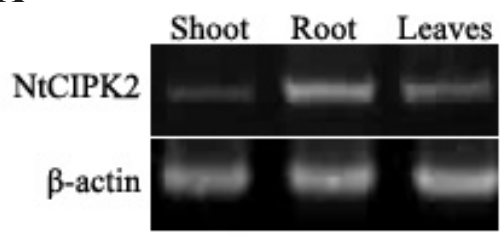

B

\begin{tabular}{|c|c|c|c|c|c|}
\hline & Control & Cold & PEG & Heat & $\mathrm{NaCl}$ \\
\hline NtCIPK2 & & 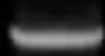 & 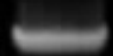 & cita & 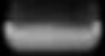 \\
\hline
\end{tabular}

Figure 4. Expression patterns of NtCIPK2 in Nitraria tangutorum. A. RT-PCR analysis in various organs. B. expression level of NtCIPK2 under abiotic stress. $\beta$-actin was used as an internal control.

\section{Overexpression of NtCIPK2 in E. coli}

Vectors were confirmed to have been transformed into Rosetta cells by restriction 
enzyme digestion and PCR analysis (data not shown). The supernatants of sonicated cell lysates from induced cells were examined by SDS-PAGE. The deduced size of the proteins from $\mathrm{RO} / \mathrm{pGEX}$ and RO/NtCIPK2 cells was approximately 26.0 and $76.6 \mathrm{kDa}$, respectively. We detected GST at $-26.0 \mathrm{kDa}$ in RO/pGEX cells, and the GST-NtCIPK2 fusion protein at -76.6 $\mathrm{kDa}$ in RO/NtCIPK2 cells (Figure 5, lanes 2 and 4, arrowheads). These results indicate that the E. coli overexpression system worked well, and that the fusion proteins were expressed effectively and were soluble.

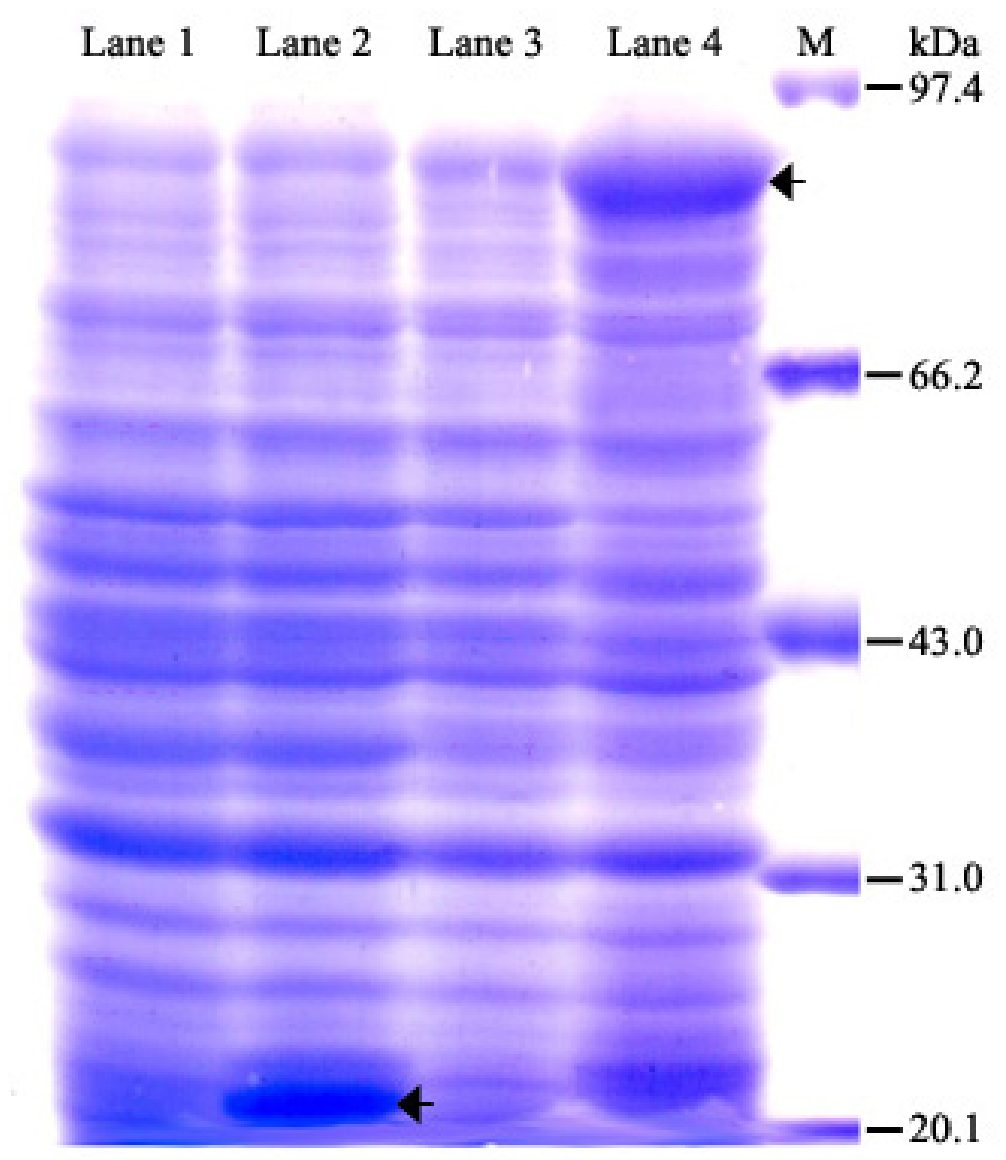

Figure 5. SDS-PAGE (12\%) analysis of fusion protein in Escherichia coli. Lane $M=$ protein marker; lane 1 $=$ supernatant of RO/pGEX cells without IPTG induction; lane $2=$ supernatant of RO/pGEX cells with IPTG induction; lane 3 = supernatant of RO/NtCIPK2 cells without IPTG induction; lane $4=$ supernatant of RO/NtCIPK2 cells with IPTG induction.

\section{Functional analysis of $\mathrm{NtCIPK2}$}

The effects of various stresses on the growth of RO/NtCIPK2 and the control strain were examined. The growth curve assay indicated no apparent distinction between the 2 strains in the absence of stress (data not shown). In contrast, RO/NtCIPK2 cells grew better in all 
treatments compared to the control cells (Figure 6). Under heat stress, the $\mathrm{A}_{600}$ value of RO/ $\mathrm{NtCIPK} 2$ cells was elevated $(\mathrm{P}<0.01)$ after $6 \mathrm{~h}$ compared to the control cells. For all other treatments, the bacterial growth was similar to $4 \mathrm{~h}$ in both cell types, after which growth improved significantly $(\mathrm{P}<0.01)$ in $\mathrm{RO} / \mathrm{NtCIPK} 2$ cells versus the control cells. The data indicate that the overexpression of NtCIPK2 improves the degree of tolerance of E. coli to abiotic stress.
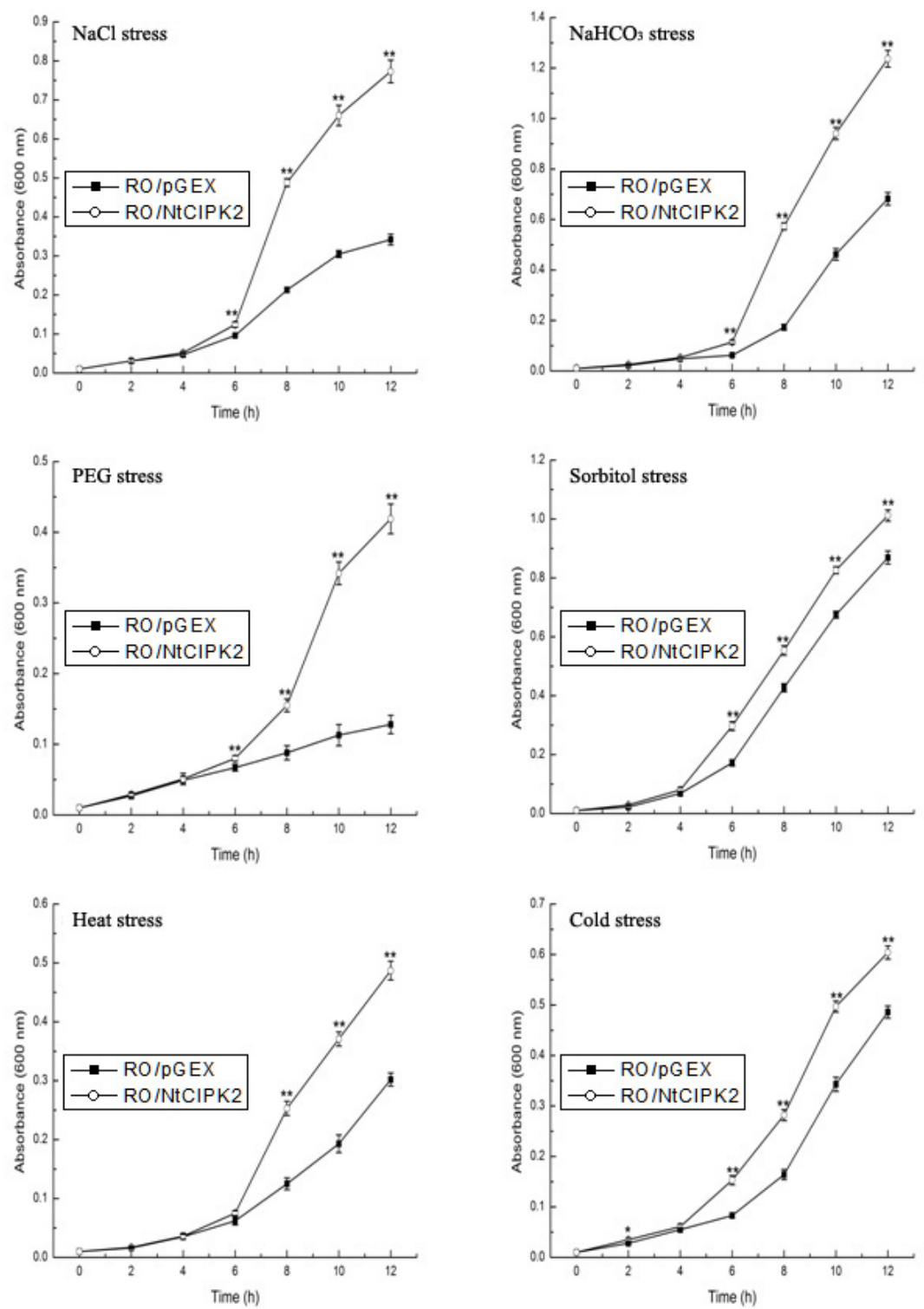

Figure 6. Growth curves of RO/pGEX (control) cells and RO/NtCIPK2 cells in liquid medium under various treatments. The average $\mathrm{A}_{600}$ and standard deviations were based on 3 independent experiments; statistical analysis was performed using ANOVA; $* \mathrm{P}<0.05$ and $* * \mathrm{P}<0.01$ indicate significant differences. 


\section{DISCUSSION}

Despite increasing evidence about the critical functions of CIPKS in mediating the stress responses of various plant species, there is a paucity of data about CIPKs from halophytes. In this study, we examined NtCIPK2, which is a member of the CIPK family in the halophyte shrub $N$. tangutorum. Like other CIPKs, NtCIPK2 bears a 2-domain structure that contains an N-terminal kinase domain and a C-terminal-regulatory domain. By mutational analysis, constitutively active forms of CIPKs might be generated by the substitution of 1 of 3 conserved residues in the N-terminal domain (Ser, Thr, and Tyr) to Asp (Guo et al., 2001; Gao et al., 2012). These residues are also found in NtCIPK2, indicating that the activity of NtCIPK2 may be improved by site-directed mutagenesis.

Several recent studies have demonstrated that CIPK2 is involved in various stress-signaling processes. Zea mays CIPK2 is upregulated by salt, PEG, cold, and heat; however, its expression differs between the leaves and the root (Chen et al., 2011). In the halophyte Hordeum brevisubulatum, $H b C I P K 2$ is strongly induced in the root and shoot by high salinity, drought, and ABA treatment. Furthermore, the overexpression of HbCIPK2 in Arabidopsis improves its tolerance to salt and osmotic stress (Li et al., 2012). In this study, to better understand the mechanism by which NtCIPK2 is regulated, an upstream fragment of the start codon was amplified. In silico analysis identified the presence of many stress-related cis-regulatory elements, such as MYB, MYC, W-BOX, LTR, and HSE, which were located in the promoter region of NtCIPK2. These elements are binding sites of transcription factors that might mediate responses to heat, cold, salinity, injury, and dehydration. We also identified ABRE in the NtCIPK2 promoter, which indicates that the expression of NtCIPK2 is ABA-dependent. Based on our promoter analysis, we selected 4 treatments $\left(\mathrm{NaCl}, \mathrm{PEG}, 50^{\circ} \mathrm{C}\right.$, and $\left.4^{\circ} \mathrm{C}\right)$ to examine the expression patterns of $N t C I P K 2$ under stress conditions. Like ZmCIPK2 and HbCIPK2, NtCIPK2 was ubiquitously expressed in all tested organs, and was induced by salinity, drought, heat, and cold stress.

As in model plant systems and several yeast mutants, E. coli may be used to validate the function of plant stress tolerance genes (Gupta et al., 2010; Song and Ahn, 2011; Chaturvedi et al., 2012). The overexpression of Lycoris radiata $S$-adenosylmethionine synthetase confers growth advantages to an engineered E. coli strain at high salt concentrations (Li et al., 2013). SbSI-1 is a novel salt-inducible gene in the halophyte Salicornia brachiata that increases tolerance to salt and desiccation in E. coli (Yadav et al., 2012). In our study, the growth rate of $\mathrm{RO} / \mathrm{NtCIPK} 2$ cells was better than control cells under salinity, drought, alkali, heat, cold, and high osmolyte stress. $N$. tangutorum primarily grows in deserts and salinity deserts, such as the Eastern Alxa-Western Ordos area, which is characterized by high soil salinity, hyperdrought, and extreme temperatures. Our results indicate that NtCIPK2 mediates stress tolerance in relation to the functioning and adaptability of $N$. tangutorum to hostile environments.

In conclusion, we cloned a novel CIPK2 gene in $N$. tangutorum, and analyzed its function. $N t C I P K 2$ was upregulated under abiotic stress, and conferred stress tolerance in $E$. coli. Thus, NtCIPK2 could potentially be used to improve the stress tolerance of crops and herbs through genetic transformation.

\section{ACKNOWLEDGMENTS}

Research supported by the National Natural Science Foundation of China (\#31140020) 
and the Natural Science Foundation Key Project of Inner Mongolia Autonomous Region (\#2012ZD05).

\section{REFERENCES}

Albrecht V, Ritz O, Linder S, Harter K, et al. (2001). The NAF domain defines a novel protein-protein interaction module conserved in $\mathrm{Ca}^{2+}$-regulated kinases. EMBO J. 20: 1051-1063.

Batistic O and Kudla J (2004). Integration and channeling of calcium signaling through the CBL calcium sensor/CIPK protein kinase network. Planta 219: 915-924.

Chaturvedi AK, Mishra A, Tiwari V and Jha B (2012). Cloning and transcript analysis of type 2 metallothionein gene (SbMT-2) from extreme halophyte Salicornia brachiata and its heterologous expression in E. coli. Gene 499: 280-287.

Chen L, Ren F, Zhou L, Wang QQ, et al. (2012). The Brassica napus calcineurin B-like 1/CBL-interacting protein kinase 6 (CBL1/CIPK6) component is involved in the plant response to abiotic stress and ABA signalling. J. Exp. Bot. 63: 6211-6222.

Chen X, Gu Z, Xin D, Hao L, et al. (2011). Identification and characterization of putative CIPK genes in maize. J. Genet. Genomics 38: 77-87.

Cheng NH, Pittman JK, Zhu JK and Hirschi KD (2004). The protein kinase SOS2 activates the Arabidopsis $\mathrm{H}^{+} / \mathrm{Ca}^{2+}$ antiporter CAX1 to integrate calcium transport and salt tolerance. J. Biol. Chem. 279: 2922-2926.

D’Angelo C, Weinl S, Batistic O, Pandey GK, et al. (2006). Alternative complex formation of the Ca-regulated protein kinase CIPK1 controls abscisic acid-dependent and independent stress responses in Arabidopsis. Plant J. 48: 857-872.

Gao P, Kolenovsky A, Cui YH, Cutler AJ, et al. (2012). Expression, purification and analysis of an Arabidopsis recombinant CBL-interacting protein kinase 3 (CIPK3) and its constitutively active form. Protein Expres. Purif. 86: 45-52.

Gong D, Gong Z, Guo Y, Chen X, et al. (2002). Biochemical and functional characterization of PKS11, a novel Arabidopsis protein kinase. J. Biol. Chem. 277: 28340-28350.

Guo Y, Halfter U, Ishitani M and Zhu JK (2001). Molecular characterization of functional domains in the protein kinase SOS2 that is required for plant salt tolerance. Plant Cell 13: 1383-1400.

Gupta K, Agarwal PK, Reddy MK and Jha B (2010). SbDREB2A, an A-2 type DREB transcription factor from extreme halophyte Salicornia brachiata confers abiotic stress tolerance in Escherichia coli. Plant Cell Rep. 29: 1131-1137.

Hu DG, Li M, Luo H, Dong QL, et al. (2012). Molecular cloning and functional characterization of MdSOS2 reveals its involvement in salt tolerance in apple callus and Arabidopsis. Plant Cell Rep. 31: 713-722.

Huang C, Ding S, Zhang H, Du H, et al. (2011). CIPK7 is involved in cold response by interacting with CBL1 in Arabidopsis thaliana. Plant Sci. 181: 57-64.

Huertas R, Olias R, Eljakaoui Z, Galvez FJ, et al. (2012). Overexpression of SISOS2 (SlCIPK24) confers salt tolerance to transgenic tomato. Plant Cell Environ. 35: 1467-1482.

Imamura M, Yuasa T, Takahashi T, Nakamura N, et al. (2008). Isolation and characterization of a cDNA coding cowpea [Vigna unguiculata (L.) Walp.] calcineurin B-like protein-interacting protein kinase, VuCIPK1. Plant Biotechnol. 25: 437-445.

Kim KN, Cheong YH, Gupta R and Luan S (2000). Interaction specificity of Arabidopsis calcineurine B-like calcium sensor and their target kinases. Plant Physiol. 124: 1844-1853.

Kim KN, Cheong YH, Grant JJ, Pandey GK, et al. (2003). CIPK3, a calcium sensor-associated protein kinase that regulates abscisic acid and cold signal transduction in Arabidopsis. Plant Cell 15: 411-423.

Knight H (2000). Calcium signaling during abiotic stress in plants. Int. Rev. Cytol. 195: 269-324.

Kolukisaoglu U, Weinl S, Blazevic D, Batistic O, et al. (2004). Calcium sensors and their interacting protein kinases: genomics of the Arabidopsis and rice CBL-CIPK signaling networks. Plant Physiol. 134: 43-58.

Li B (1990). Natural Resources and Environment Study of Inner Mongolia Ordos Plateau. Science Press, Beijing.

Li LB, Zhang YR, Liu KC, Ni ZF, et al. (2010). Identification and bioinformatics analysis of SnRK2 and CIPK family genes in sorghum. Agr. Sci. China 9: 19-30.

Li RF, Zhang JW, Wei JH, Wang HZ, et al. (2009). Functions and mechanisms of the CBL-CIPK signaling system in plant response to abiotic stress. Prog. Nat. Sci. 19: 667-676.

Li RF, Zhang JW, Wu GY, Wang HZ, et al. (2012). HbCIPK2, a novel CBL-interacting protein kinase from halophyte Hordeum brevisubulatum, confers salt and osmotic stress tolerance. Plant Cell Environ. 35: 1582-1600.

Li XD, Xia B, Wang R, Xu S, et al. (2013). Molecular cloning and characterization of S-adenosylmethionine synthetase gene from Lycoris radiata. Mol. Biol. Rep. 40: 1255-1263.

Genetics and Molecular Research 13 (3): 4716-4728 (2014)

CFUNPEC-RP www.funpecrp.com.br 
Liu LL, Ren HM, Chen LQ, Wang Y, et al. (2013). A protein kinase, calcineurin B-like protein-interacting protein kinase 9, interacts with calcium sensor calcineurin B-like protein 3 and regulates potassium homeostasis under low-potassium stress in Arabidopsis. Plant Physiol. 161: 266-277.

Pandey GK, Cheong YH, Kim BG, Grant JJ, et al. (2007). CIPK9: a calcium sensor-interacting protein kinase required for low-potassium tolerance in Arabidopsis. Cell Res. 17: 411-421.

Qiu QS, Guo Y, Quintero FJ, Pardo JM, et al. (2004). Regulation of vacuolar $\mathrm{Na}^{+} / \mathrm{H}^{+}$exchange in Arabidopsis thaliana by the salt-overly-sensitive (SOS) pathway. J. Biol. Chem. 279: 207-215.

Sánchez-Barrena MJ, Martinez-Ripoll M, Zhu JK and Albert A (2005). The structure of the Arabidopsis thaliana SOS3: molecular mechanism of sensing calcium for salt stress response. J. Mol. Biol. 345: 1253-1264.

Sanders D, Pelloux J, Brownlee C and Harper JF (2002). Calcium at the crossroads of signaling. Plant Cell (Suppl 14): S401-S417.

Song NH and Ahn YJ (2011). DcHsp17.7, a small heat shock protein in carrot, is tissue-specifically expressed under salt stress and confers tolerance to salinity. New Biotechnol. 28: 698-704.

Tai FJ, Wang Q, Yuan ZL, Yuan ZH, et al. (2013). Characterization of five CIPK genes expressions in maize under water stress. Acta Physiol. Plant 35: Doi: 10.1007/s11738-012-1197-2.

Tan GH, Gao Y, Shi M, Zhang XY, et al. (2005). Site Finding-PCR: a simple and efficient PCR method for chromosome walking. Nucleic Acids Res. 33: e122.

Tripathi V, Parasuraman B, Laxmi A and Chattopadhyay D (2009). CIPK6, a CBL-interacting protein kinase is required for development and salt tolerance in plants. Plant J. 144: 1416-1428.

Wang HL, Suo YR, Wang XY, Li YL, et al. (2007). Extraction of Nitraria tangutorum seed oil by supercritical carbon dioxide and determination of free fatty acids by HPLC/APCI/MS with fluorescence detection. Sep. Purif. Technol. 56: 371-377.

Wang RK, Li LL, Cao ZH, Zhao Q, et al. (2012). Molecular cloning and functional characterization of a novel apple MdCIPK6L gene reveals its involvement in multiple abiotic stress tolerance in transgenic plants. Plant Mol. Biol. 79: 123-135.

Weinl S and Kudla J (2009). The CBL-CIPK $\mathrm{Ca}^{2+}$-decoding signaling network: function and perspectives. New Phytol. 184: 517-528.

Xiang Y, Huang YM and Xiong LZ (2007). Characterization of stress-responsive CIPK genes in rice for stress tolerance improvement. Plant Physiol. 144: 1416-1428.

Xu J, Li HD, Chen LQ, Wang Y, et al. (2006). A protein kinase, interacting with two calcineurin B-like proteins, regulates $\mathrm{K}^{+}$transporter AKT1 in Arabidopsis. Cell 125: 1347-1360.

Yadav NS, Rashmi D, Singh D, Agarwal PK, et al. (2012). A novel salt-inducible gene SbSI-1 from Salicornia brachiata confers salt and desiccation tolerance in E. coli. Mol. Biol. Rep. 39: 1943-1948.

Yang YL, Wei XL, Shi RX, Fan Q, et al. (2010). Salinity-induced physiological modification in the callus from halophyte Nitraria tangutorum Bobr. J. Plant Growth Regul. 29: 465-476.

Yang YL, Yang F, Li XN, Shi RX, et al. (2013). Signal regulation of proline metabolism in callus of the halophyte Nitraria tangutorum Bobr. grown under salinity stress. Plant Cell Tiss. Org. 112: 33-42.

Yu YH, Xia XL, Yin WL and Zhang HC (2007). Comparative genomic analysis of CIPK gene family in Arabidopsis and Populus. Plant Growth Regul. 52: 101-110.

Zhao J, Sun Z, Zheng J, Guo X, et al. (2009). Cloning and characterization of a novel CBL-interacting protein kinase from maize. Plant Mol. Biol. 69: 661-674.

Zheng J, Li H, Ding CX, Suo YR, et al. (2011). Anthocyanins composition and antioxidant activity of two major wild Nitraria tangutorum Bobr. variations from Qinghai-Tibet Plateau. Food Res Int. 44: 2041-2046.

Genetics and Molecular Research 13 (3): 4716-4728 (2014) 